\title{
Bioethics and the Law in Familial Cancer, a Practical Approach
}

\author{
Azua-Romeo Javier \\ Department of Anatomy and Histology, University of Zaragoza, Zaragoza, Spain. \\ Email: javierazua@hotmail.com
}

Received December $27^{\text {th }}$, 2011; revised January 31 ${ }^{\text {st }}$, 2012; accepted February $24^{\text {th }}, 2012$

\begin{abstract}
Hereditary cancers are the result of germline mutations in specific genes that increase susceptibility to cancer. This susceptibility is spread among family members according to different patterns of inheritance. Inherited susceptibility to cancer, does not imply the certainty of developing it in all cases. In this sense many questions have come out in recent years, not only in the medical sense but also in the field of bioethics and the law.
\end{abstract}

Keywords: Cancer; Hereditary; Ethics; Law

\section{Clinical Issues Related to Familial Cancer}

\subsection{Main Characteristics of Familial Cancer}

Cancers are multifactorial diseases due to a combination of genetic and environmental factors. A small proportion, it is estimated that between $5 \%$ and $10 \%$ of all tumors, has a familial or hereditary features. Hereditary cancers are the result of germline mutations in specific genes that increase susceptibility to cancer. This susceptibility is spread among family members according to different patterns of inheritance. Inherited susceptibility to cancer, does not imply the certainty of developing it in all cases.

In recent years we have identified about 40 genes involved in many other syndromes of inherited predisposition to cancer and have developed techniques for characterization of mutations.

The identification of families with hereditary cancer is really important since its members can benefit from effective measures regarding early detection, and to establish preventive strategies to decrease the possibility of developing certain tumours. In families with hereditary cancer it is common to observe several cases among its members, usually suffering from the same type that appears in a generation and the next. These cancers appear at an early age, in the most common syndromes, such as breast, ovarian and colorectal cancer, at 40 and 50 years of age.

\subsection{Genetic Counseling}

Inheritable and genetic diseases can appear in one family, a circumstance that brings new responsibilities for the health professional. Attention to families with hereditary cancer requires genetic counselling specialists who will evaluate individual risk for each family member, and appropriate genetic determinations. Through genetic counselling, patients with hereditary predisposition to cancer receive information on: 1) the probability of malignancy, 2) the probability of transmitting to their offspring's predisposition to cancer and the likelihood of states to develop malignancies, and 3) prognosis, strategies for early detection and more appropriate therapeutic approach.

Psychological support seems very interesting previous to the establishment of therapeutic/preventive measures.

The specific genetic testing is not possible in all cases, as there are entities in which we not even know the gene responsible. The patient should be informed which genetic studies are most appropriate in his case and what consequences would be to expect positive or negative. [1-3].

\subsection{Preventive Measures}

Another aspect of hereditary cancer that has great impact on families is the monitoring of individuals at risk [4-6]. In clinical practice today, there are three main tools to handle these situations: 1 ) periodic medical surveillance, 2) chemoprevention, and 3) prophylactic surgery [7-9]. The tool used is the medical surveillance, especially in the service of Medical Oncology. There are consensus recommendations for care of individuals with the most common syndromes with predisposition to cancer, including Hereditary Breast and Ovarian Cancer (HBOC), the Lynch and Cowden syndrome, Familial Adenomatous Polyposis (FAP), Endocrine Multiple Neoplasm or 
neurofibromatosis. For its part, chemoprevention (based on the administration of several drugs) in most situations is still under investigation. Prophylactic surgery (for example: countralateral breast resection in case of breast cancer in young patients) allows the primary prevention and has application in FAP, HBOC and to a lesser extent in the Lynch syndrome.

\subsection{Medical Monitoring}

When there is not known genetic defect responsible for cancer in the family, all people at risk (usually first-degree relatives of those affected), will be under close medical supervision. The knowledge of the specific genetic mutations allows restricted follow up to the family members found to be carriers of such alteration.

Hereditary cancer has very different characteristics from sporadic cancer:

- Early age of onset.

- Multifocality or bilateral lesions.

- Appearance of several primary tumours in the same individual.

- Family history of the same neoplasm.

- High incidence of cancer within the same family.

- Association of widespread body tumours or asymmetric overgrowth, dysmorphic features, congenital malformations or mental retardation.

Any of these situations should alert the clinician to the possibility of being faced with a case of hereditary cancer and the appropriateness of referring for genetic counselling to the family.

The American Society of Clinical Oncology (ASCO) recommends that familiar genetic determination in hereditary cancer shall be offered when:

- The patient is diagnosed with cancer at a younger age than expected, and/or his family have a significant accumulation of tumours.

- The results of genetic testing, can be interpreted reliably.

- The results may have an impact on the therapeutic management of the patient or his family.

\section{A Clinical Case}

\subsection{Description of Facts}

Family with definite criteria of familial gastric cancer, in which there are several members under 18 years of age:

- Father of 42 years of age who developed a gastric cancer, and who suffered total gastrectomy, remaining alive at this time. Gastric tissue and blood available for genetic studies. Which yields positive results to genetic alterations in $\mathrm{CDH}-1$ gen, concordant with hereditary gastric cancer.

- Father's Grandfather died at the age of 44 due colo- rectal cancer. Neither available tissue nor blood samples.

- Father's Brother, 38 years of age, asymptomatic, absence of lesions through gastroscopy. Genetic analysis performed using blood samples shows same genetic alterations in CDH1 gene.

- Two sons of 14 and 12, totally asymptomatic. Blood samples indicate that only one of them is a carrier of genetic alterations related to gastric hereditary cancer.

- Mother free of disease with no familiar history of cancer.

\subsection{Questions Raised for Discussion}

- In case of definitive diagnosis of hereditary cancer, shall we inform all the patient relatives? or should we let the patient himself warn his relatives?

- Whether the target relative declines to be studied what can we do, knowing the high probabilities to be a carrier of a hereditary cancer? And if the relative has children, should we inform the other partner?

- Whether aggressive treatment is decided (prophylactic gastrectomy), who has to give written consent, when the patient is 1 ) a competent adult; 2) a child?

\section{REFERENCES}

[1] K. Offit and P. Thom, "Ethicolegal Aspects of Cancer Genetics,” Cancer Treatment Research, Vol. 155, 2010, pp. 1-14. doi:10.1007/978-1-4419-6033-7 1

[2] S. L. Manne, N. J. Meropol, D. S. Weinberg, H. Vig, Z. A.-K. Catts, C. Manning, E. Ross, K. Shannon and D. C. Chung, "Facilitating Informed Decisions Regarding Microsatellite Instability Testing among High-Risk Individuals Diagnosed with Colorectal Cancer," Journal of Clinical Oncology, Vol. 28, No. 8, 2010, pp. 1366-1372. doi:10.1200/JCO.2009.25.0399

[3] M. Aronson, "Genetic Counselling for Hereditary Colorectal Cancer: Ethical, Legal and Psychosocial Issues," Surgical Oncology Clinics of North America, Vol. 18, No. 4, 2009, pp. 669-685. doi:10.1016/j.soc.2009.07.001

[4] J. Kountouras, C. Zavos and D. Chatzopoulos, "New Concepts of Molecular Biology on Gastric Carcinogenesis,” Hepato-Gastroenterology, Vol. 52, No. 64, 2005, pp. 1305-1312.

[5] A. Romiti, A. Zullo, S. Tomao, C. Hassan, I Sarcina, V. D. Francesco, E. Ierardi, F. Tomao, A. Vecchione and S. Morini, "Gastric Mucosa Alterations in First-Degree Relatives of Gastric Cancer Patients,” Anticancer Reseach, Vol. 25, No. 3c, 2005, pp. 2567-2572.

[6] D. G. Huntsman, F. Carneiro, F. R. Lewis, et al., "Early Gastric Cancer in Young, Asymptomatic Carriers of GermLine E-Cadherin Mutations,” The New England Journal of Medicine, Vol. 344, No. 25, 2001, pp. 1904- 1909.

[7] F. R. Lewis, J. D. Mellinger, A. Hayashi, et al., "Prophylactic Total Gastrectomy for Familial Gastric Cancer," Surgery, Vol. 130, No. 4, 2001, pp. 612-617. 
doi:10.1067/msy.2001.117099

[8] C. Caldas, F. Carneiro, H. Lynch, et al., "Familial Gastric Cancer: Overview and Guidelines for Management," Journal of Medical Genetics, Vol. 36, No. 12, 1999, pp. 873-880.

[9] G. Suriano, S. Yew, P. Ferreira, et al., "Characterization of a Recurrent Germ Line Mutation of the E-Cadherin gene: Implications for Genetic Testing and Clinical Management," Clinical Cancer Research, Vol. 11, No. 15, 2005, pp. 5401-5409.

doi:10.1158/1078-0432.CCR-05-0247 